OPEN ACCESS

Edited by:

Angelo Labate,

University of Catanzaro, Italy

Reviewed by:

Edoardo Ferlazzo,

University of Catanzaro, Italy

Elena Gardella,

University of Southern

Denmark, Denmark

*Correspondence:

Francesca Bisull

francesca.bisulli@unibo.it

Specialty section:

This article was submitted to

Epilepsy,

a section of the journal

Frontiers in Neurology

Received: 02 November 2021

Accepted: 31 December 2021

Published: 27 January 2022

Citation:

Furia A, Licchetta L, Muccioli L,

Ferri L, Mostacci B, Mazzoni S,

Menghi V, Minardi R, Tinuper $P$ and

Bisulli F (2022) Epilepsy With Auditory

Features: From Etiology to Treatment.

Front. Neurol. 12:807939

doi: 10.3389/fneur.2021.807939

\section{Epilepsy With Auditory Features: From Etiology to Treatment}

\author{
Alessandro Furia ${ }^{1}$, Laura Licchetta ${ }^{2}$, Lorenzo Muccioli ${ }^{1}$, Lorenzo Ferri ${ }^{1,2}$, \\ Barbara Mostacci ${ }^{2}$, Stefania Mazzoni ${ }^{2}$, Veronica Menghi ${ }^{1}$, Raffaella Minardi ${ }^{2}$, \\ Paolo Tinuper ${ }^{1,2}$ and Francesca Bisulli ${ }^{1,2 *}$
}

${ }^{1}$ Department of Biomedical and NeuroMotor Sciences, Alma Mater Studiorum-University of Bologna, Bologna, Italy, ${ }^{2}$ Istituto di Ricerca e Cura a Carattere Scientifico (IRCCS) Istituto delle Scienze Neurologiche di Bologna, Reference Center for Rare and Complex Epilepsies, Bologna, Italy

Epilepsy with auditory features (EAF) is a focal epilepsy belonging to the focal epileptic syndromes with onset at variable age according to the new ILAE Classification. It is characterized by seizures with auditory aura or receptive aphasia suggesting a lateral temporal lobe involvement of the epileptic discharge. Etiological factors underlying EAF are largely unknown. In the familial cases with an autosomal dominant pattern of inheritance several genes have been involved, among which the first discovered, LG/1, was thought to be predominant. However, increasing evidence now points to a multifactorial etiology, as familial and sporadic EAF share a virtually identical electro-clinical characterization and only a few have a documented genetic etiology. Patients with EAF usually have an unremarkable neurological examination and a good response to antiseizure medications. However, it must be underscored that total remission might be lower than expected and that treatment withdrawal might lead to relapses. Thus, a proper understanding of this condition is in order for better patient treatment and counseling. Further studies are still required to further characterize the many facets of EAF.

Keywords: auditory hallucinations, epilepsy, DEPDC5, mTOR, LGI1, aphasic seizures

\section{INTRODUCTION}

Epilepsy with auditory features (EAF) is a focal epilepsy included in the group of focal syndromes with onset at variable age in the new ILAE Classification, besides focal epilepsy with variable foci (FFEAF) and sleep-related hypermotor epilepsy (SHE), with which it shares some genetic aspects (1). The new simplified nomenclature aims to overcome the previous, possibly misleading, use of two different names for the same syndrome, namely autosomal dominant lateral temporal lobe epilepsy (ADLTLE) and autosomal dominant partial epilepsy with auditory features (ADPEAF), and to acknowledge this entity also in a non-familial context/pattern.

EAF phenotype is characterized by seizures with auditory symptoms or aphasia, suggesting a lateral temporal lobe involvement.

Auditory symptoms have long been associated with epilepsy, with the first description of such presentation dating as early as 1,883 (2). More generally, auditory hallucinations may be classified as simple (hearing a monotone sound such as humming or buzzing as in tinnitus) and complex (hearing voices or music), in which case they can be defined as auditory verbal hallucinations (3). On this basis, it has also been proposed that several historical figures were affected by epilepsy with auditory hallucinations $(4,5)$, including Fyodor Dostoevsky, whose known epilepsy might have 
first manifested in childhood with verbal hallucinations, as illustrated in his autobiographical work "The Peasant Marey" (5).

Aside from reasons of historical interest, recognizing the epileptic origin of auditory phenomena/symptoms is key for both the neurologist and other clinicians. Quite commonly, auditory features in epilepsy are misdiagnosed as psychiatric disease, most often schizophrenia, as shown in several reports (6-8).

Even within the field of epileptology, appreciating the clinical features of EAF, an entity whose prevalence might be higher than expected, is paramount to a proper diagnosis and adequate treatment.

Additionally, recognizing the propensity of lateral temporal seizures to evolve into focal to bilateral tonic-clonic ones helps avoid the pitfall of misdiagnosing focal epilepsy as an idiopathic generalized entity, with consequent therapeutic implications.

\section{HISTORY AND NOMENCLATURE FROM 1995 TO 2021}

In 1995, on the same Nature Genetics volume in which a genetic basis for SHE was first described (9) Ottman et al. published a study in which they identified a locus on chromosome $10 \mathrm{q}$ associated with focal epilepsy in 11 members of a large family (10). This entity showed autosomal dominant transmission with high penetrance. Of the 11 members affected, 55\% reported auditory symptoms: all had focal to bilateral tonic-clonic seizures. Despite being identified only relatively recently, EAF stands as an example of how a paradigmatic shift allowed to focus on the genetic basis of focal, and not only generalized, epilepsy.

This first observation was then corroborated in 1999 by Poza et al., who described 19 individuals from a Basque family suffering from a similar form of focal epilepsy, even in this case linked to chromosome 10q (11). However, compared to the earlier study, the members also showed other sensory symptoms, such as visual hallucinations. A new term was proposed for such epilepsy, autosomal dominant lateral temporal lobe epilepsy (ADLTE), to highlight the supposed anatomical origin instead of mesial temporal epilepsy.

Subsequently, Winawer et al. further elaborated their clinical description of the 1999 family (12). In particular, the Authors introduced an alternative name, i.e., autosomal dominant partial epilepsy with auditory features (ADPEAF), to emphasize the high prevalence of ictal auditory symptoms.

A decisive turn in the characterization of ADLTE/ADPEAF was made in 2002, when Kalachicov et al. identified the first causative gene of ADLTE, LGI1, on chromosome 10q24. In particular, they identified five different pathogenic LGI1 variants, of which three were frameshift, one was missense and the last one was a splice site variant (13). LGI1 mutations were also associated to temporal lobe epilepsy with aphasic seizures (14), demonstrating that other ictal manifestations are to be considered in the phenotypic spectrum of temporal lobe epilepsy with auditory features. The putative role of LGI1 in both normal and disease states will be discussed in the sections below.

In 2015, causative variants of a second gene, RELN, were found in seven families (15), followed by identifications of other genes, including DEPDC5, MICAL-1, CNTNAP2, and SCN1A (16-18).

In 2021, the Nosology and Definition Task Force of the ILAE, by introducing position papers for definition and new nomenclature of epileptic syndromes (1), proposed the term Epilepsy with Auditory Features (EAF) to encompass both ADLTE and ADPEAF, also defining the inherited form as Familial EAF (FEAF).

\section{CLINICAL FEATURES AND INVESTIGATIVE FINDINGS}

Since the 1989 ILAE classification of epilepsies and epileptic syndromes (19), temporal lobe epilepsy has been divided into mesial and lateral forms: the most salient features distinguishing the two are shown in Table 1. It can be noticed that auditory symptoms are a prominent feature of lateral temporal epilepsy, therefore being of great clinical help.

The clinical features of EAF are summarized in Table 2.

Considering that isolated auditory symptoms, especially when these are simple, might not be adequately recognized as pertaining to a disease and in particular epilepsy, a precise estimate of the incidence of EAF is currently not available.

Several studies have investigated the clinical presentation of both sporadic and familial EAF (22-24): notably, it appears that there are no significant clinical differences between the two forms (22).

It is now understood that EAF is an epileptic syndrome whose onset is not age-related although it is more frequent in the second and third decade of life.

Patients do not show abnormalities at the physical or neurological exam and in their personal history of birth and development.

As the name suggests, the key feature of EAF consists of focal aware sensory seizures with auditory symptoms, which can be both simple or complex. Patients can also present with focal aware cognitive seizures, i.e., receptive aphasia. Oher sensory seizures (most often visual) may also occur. Focal to bilateral tonic-clonic or focal impaired awareness seizures are possible. Most importantly, these might be the first seizures to be noticed and reported. These seizures frequently occur during sleep and the focal signs, namely auditory features, may be easily missed, possibly leading to an idiopathic generalized epilepsy misdiagnosis (1).

The most important entity in differential diagnosis with FEAF is FFEVF, which can also present with auditory seizures. However, diagnostic criteria for EAF require that all affected family members present with auditory symptoms, as opposed to isolated family members in FFEVF.

Simple and complex auditory hallucinations pertaining to other disorders (most commonly, tinnitus or schizophrenia) can be excluded by careful clinical observation and history gathering: the sound of tinnitus is far more durable than the seizure of EAF, while schizophrenic hallucinations are quite complex and often accompanied by other features of this psychiatric disease. 
TABLE 1 | Differences between mesial and lateral temporal lobe epilepsy.

\begin{tabular}{|c|c|c|}
\hline & Mesial temporal lobe epilepsy & Lateral temporal lobe epilepsy \\
\hline Localization & $\begin{array}{l}\text { Hippocampus, parahippocampal gyrus, amygdala, entorhinal } \\
\text { cortex }\end{array}$ & Temporal neocortex \\
\hline $\begin{array}{l}\text { Seizure evolution in focal } \\
\text { to bilateral tonic-clonic }\end{array}$ & Less frequent & More frequent \\
\hline $\begin{array}{l}\text { Interictal EEG } \\
\text { abnormalities }\end{array}$ & More frequent & Less frequent \\
\hline Drug resistance & More frequent & Less frequent \\
\hline
\end{tabular}

TABLE 2 | Clinical features of EAF [adapted from (21)].

\begin{tabular}{ll}
\hline Family history & $\begin{array}{l}\text { Might be positive for febrile seizures, rarely for } \\
\text { intellectual disability or psychiatric disorders }\end{array}$ \\
Personal history & Might be positive for febrile seizures \\
Interictal EEG & $\begin{array}{l}\text { Usually normal, in some cases focal (temporal) or } \\
\text { diffuse epileptiform abnormalities }\end{array}$ \\
Seizure semiology & $\begin{array}{l}\text { Aura: might be auditory or aphasic (receptive/global } \\
\text { aphasia) }\end{array}$ \\
& $\begin{array}{l}\text { Auditory hallucinations: simple/complex } \\
\text { Focal to generalized tonic-clonic seizures are possible } \\
\text { Reflex seizures from hearing a sound could be present }\end{array}$
\end{tabular}

Interictal EEG is often unremarkable, but it may show focal temporal sharp waves or spikes (Figure 1).

Some patients with EAF may have an underlying lesion on brain MRI (Figure 2); however, in contrast to what described in early studies (23), also reporting cases associated with LGI1 mutations (24), this occurrence is infrequent (25). This notwithstanding, high-resolution MRI remains a key investigational procedure in patients with EAF in order to exclude a lesional etiology, a finding which may have remarkable therapeutic implications, notably epilepsy surgery in selected patients. In some cases, the absence of an underlying lesion might represent a false negative finding related to insufficient MRI resolution; however, a non-lesional cause should become the prime suspect. Some cases with lesional EAF may have an underlying mutation, such as GATOR1-related focal cortical dysplasia; however, as discussed below, the genetics of EAF has not been fully elucidated yet, and genotype-phenotype correlations are difficult to establish.

\section{GENETICS: FROM LGI1 TO mTOR GENES}

The genes involved with EAF are presented in Table 3. These will now be reviewed in more detail.

\section{LGI1}

Leucine-rich, glioma inactivated protein 1 (also known as epitempin) is a protein encoded by the LGI1 gene on chromosome $10 \mathrm{q} 24$. Initially discovered by Chernova and colleagues in 1998 (27), LGI1 is a predominantly neuronal protein, whose downregulation was first implicated in the pathogenesis of malignant brain neoplasms (in particular glioblastoma multiforme) as its own name suggests. Thus, its putative role was believed to be tumor suppression. However, LGI1 has been implicated in various other processes, including neuronal transmission (28) and development (29): therefore, its role remains to be fully elucidated.

Epitempin binds to its receptor, ADAM22 (part of the transmembrane ADAM metalloprotease family), forming a complex which regulates neurotransmission through inhibition of the AMPA receptor $(30,31)$.

More than 40 pathogenic variants of LGI1 have been described in EAF (30). These mutations, which all lead to loss of function, point to a likely mechanism of LGI1 haploinsufficiency in causing EAF, which would also explain the autosomal dominant pattern of inheritance. Moreover, the temporal origin of seizures and the present evidence regarding epitempin suggest that abnormal neuronal migration with the formation of dysfunctional circuits might be the underlying mechanism linking LGI1 to epilepsy (30).

Also, antibodies against epitempin disrupting the LGI1ADAM22 complex cause a rare form of autoimmune encephalitis, characterized in some cases by peculiar faciobrachial dystonic seizures (FBDS) preceding the full-fledged clinical picture of cognitive dysfunction, memory impairment, and, eventually, tonic-clonic seizures. Interestingly, FBDS respond exquisitely well to immunotherapy (32). Future research might uncover a possible relation, currently not clear, between these close but different entities. 


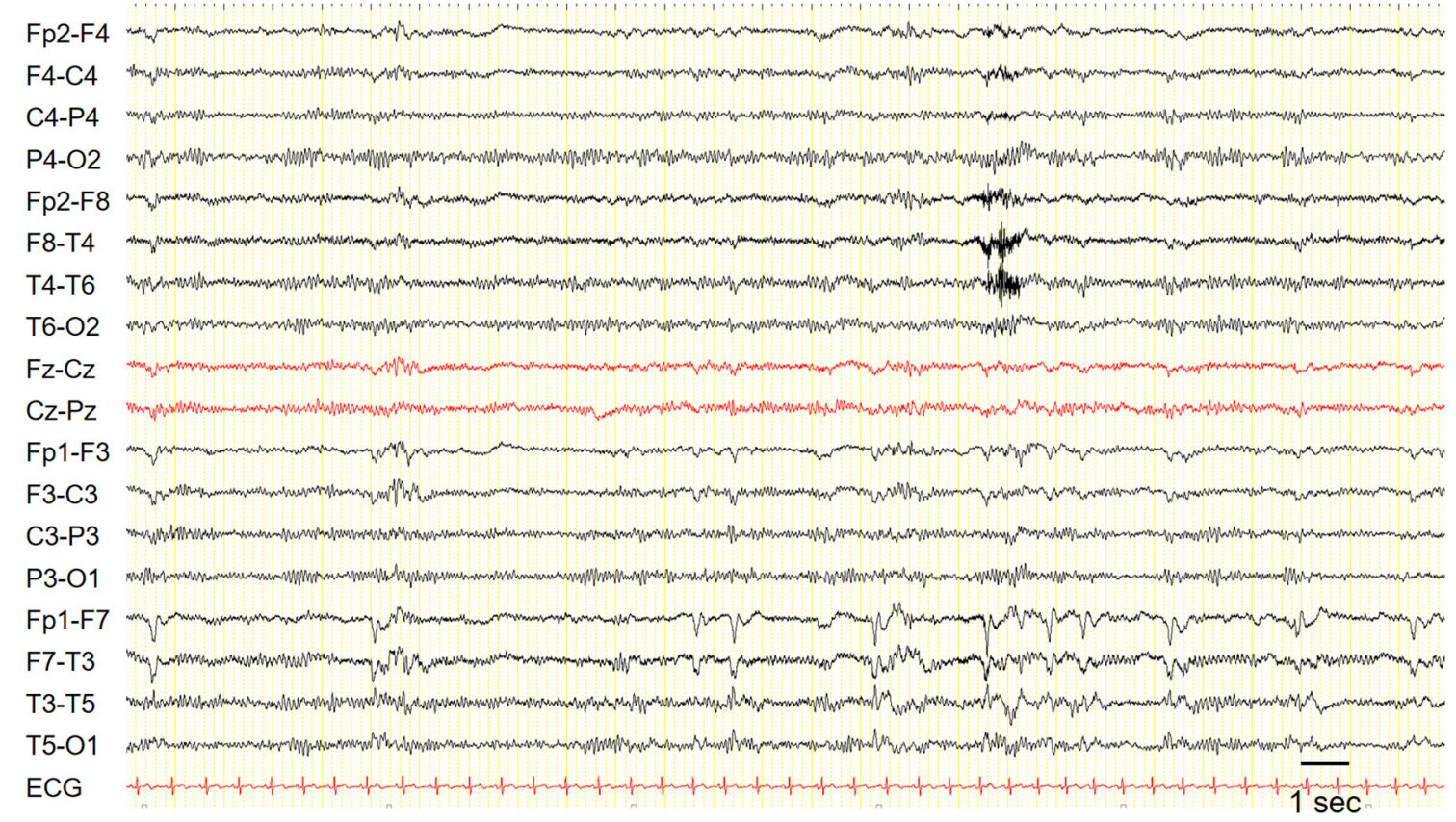

FIGURE 1 | EEG in a patient with epilepsy with auditory features showed recurrent epileptiform discharges in the left fronto-temporal region. Sensitivity: 7 uV/mm.

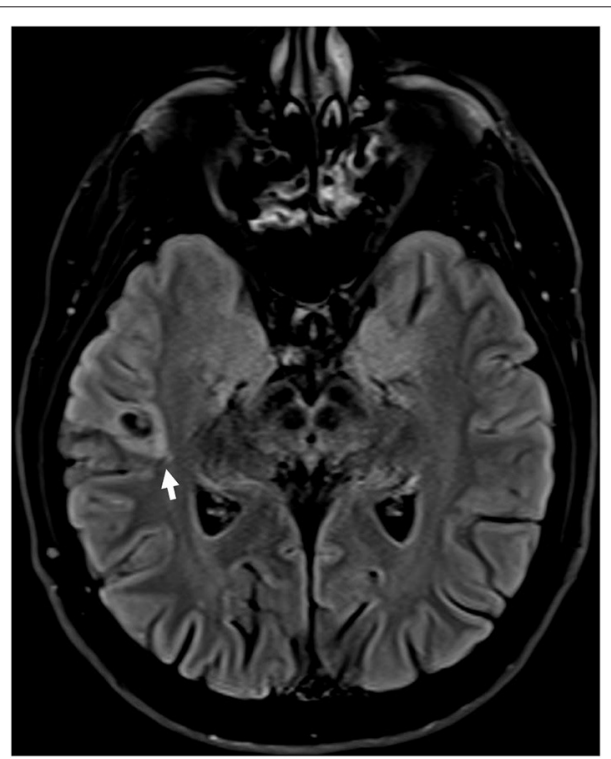

FIGURE 2 | Brain MRI (FLAIR; axial view) in a patient with drug-resistant epilepsy with auditory features showed a small hyperintense lesion in the right lateral temporal cortex surrounded by an area of signal suppression and hyperintensity of the adjacent brain tissue, consistent with a glioneuronal tumor.

\section{DEPDC5 AND THE MTOR PATHWAY}

The DEPDC5 (DEP domain containing 5, GATOR1 subcomplex subunit) gene has been associated to all the three epileptic syndromes with onset at variable age, thus comprising SHE, FFEVF and EAF, as well as mesial temporal lobe epilepsy
TABLE 3 | Genes linked to EAF.

\begin{tabular}{ll}
\hline LGI1 & Identified in 1995 \\
& Modulation of AMPA-related neurotransmission \\
RELN & Identified in 2017 \\
& Neuronal migration and plasticity \\
DEPDC5 & Identified in 2015 \\
& Role in mTOR pathway and cortical dysplasia \\
CNTNAP2 & Identified in 2018 \\
SCN1A & Encodes a neurexin protein \\
& Identified in 2019 \\
& Related to generalized epilepsy with febrile seizures \\
plus (GEFS+): associated to EAF in (26) \\
Identified in 2018 \\
Cytoskeletal regulation \\
Not currently confirmed
\end{tabular}

$(16,33)$. The GATOR1-complex is a negative controller of the mTOR (mammalian target of rapamycin) pathway, a molecular pathway fundamental in several functions key to cell survival, such as proliferation and growth (34). Developmental brain abnormalities caused by pathogenic variants in DEPDC5 and genes coding for other component of the mTOR pathway might justify its association with focal cortical dysplasia (FCD), which according to its anatomical position might cause a wide array of different DEPDC5-related focal epilepsies. However, not all individuals carrying DEPDC5 variants show FCD on brain MRI, suggesting that, in case of missense variants, brain structural lesions are caused by and additional brain somatic mutation, according to a double hit mechanism. DEPDC5-related EAF was first reported by Pippucci et al. in a family with few affected indivuals showed EAF as predominant epilepsy phenotype (16). 
It can be argued if the family studied represents instead a FFEVF pedigree, due to its small size. This notwithstanding, if the relation to EAF is established, mTOR targeting with specific drugs might be useful in the subset of patients with DEPDC5related $\mathrm{EAF}$.

\section{OTHER GENES (RELN, MICAL-1, CNTNAP2, SCN1A)}

The RELN gene on chromosome region $7 \mathrm{q} 22$ encodes reelin, a large secreted protein that modulates both neuronal migration in the embryonal stage and neuronal plasticity in adult life $(26,35)$. Reelin has also been implicated in several neurological disorders, comprising lissencephaly and Alzheimer's disease $(36,37)$. RELN mutations were recently discovered in families affected by EAF, but no differences in clinical phenotype with LGI1-related epilepsy could be found (22).

The MICAL-1 (Microtubule Associated Monooxygenase, Calponin and LIM Domain Containing 1) gene, on chromosome $6 \mathrm{q} 21$, encodes for a protein regulating the actin cytoskeleton. Likely pathogenic variants were identified in two unrelated EAF family in one study (18), but the association of the MICAL1 gene with EAF is still under scrutiny. Other described mutations include CNTNAP2 (Contactin-associated protein-like 2) and SCN1A (sodium channel, voltage-gated, type I, alpha subunit), which might point that EAF pertains to the spectrum of SCN1Arelated epilepsy $(16,38,39)$.

In conclusion, while it is commonly believed that LGI1 and RELN together make up for most of the cases of FEAF, a recent study has suggested that truly genetically determined cases are rare (21) and that etiology could be multifactorial. Therefore, along with further discoveries of new genetic mutations underlying EAF, a more precise characterization of known defects is needed, too.

\section{THERAPY}

EAF is considered a syndrome with a good response to anti-seizure medications used for focal epilepsy, such as carbamazepine in monotherapy (40). However, patients might refuse pharmacological therapy for a disease perceived as trivial, especially in milder presentations. Surgery might be employed instead in resistant cases. When misdiagnosed as idiopathic generalized epilepsy, EAF might be treated with drugs that are not optimal (i.e., phenobarbital or valproate), leading to poor response and to the risk of mislabeling a treatable epileptic syndrome as drug-resistant.

Another aspect to consider is that drug withdrawal often leads to clinical relapses, not always responding to reinitiation of treatment (40). These aspects should be stressed when discussing the therapeutic options with the patient.

\section{PROGNOSIS}

Studies concerning the prognosis of both sporadic and familial EAF are only a few and mostly consist of reports of isolated families with few individuals.
The largest cohort of EAF patients was collected in a study by our group (40), in which 123 EAF patients (mostly sporadic) were followed with a median time of 11 years, using as primary endpoint total remission (seizure-free period of more than 5 years).

The three key factors negatively affecting remission in the cohort were the age onset ( $<10$ years), complex auditory hallucinations, and focal EEG epileptiform abnormalities. A paramount aspect to be considered is the significant heterogeneity of the cohort in terms of phenotype's severity, from mild to refractory cases only referable by surgery, which might also explain why the total remission rate in this study was lower than expected $(34.1 \%)$.

\section{CONCLUSIONS}

The research field on EAF still presents several questions and challenges to be addressed. First, the actual prevalence of this syndrome must be thoroughly investigated.

Second, more work should be done on genetics, perhaps shifting the original paradigm from a disease dominated by LGI1 mutations to a multifactorial syndrome that can be stratified in different groups.

Following this direction, it could be possible in the future to separate patients into favorable groups where pharmacological treatment is useful and groups where epilepsy is strongly resistant, with surgery as the only viable option. In this sense, as evidence is still lacking, more effort should be spent on characterizing DEPDC5-related EAF and understanding the extent of mutation-associated cortical dysplasia.

In conclusion, EAF demonstrates how much progress has been made in the field of genetics in focal epilepsy, historically considered the paradigm of lesional/suspected lesional epilepsies, and how much more can be achieved in the future.

\section{AUTHOR CONTRIBUTIONS}

AF wrote the first draft of the manuscript. RM and LL reviewed the literature on the genetic aspects of EAF and provided corrections. LM, LF, and BM reviewed the manuscript concerning the section on clinical presentation, treatment, and prognosis. SM, VM, RM, PT, and FB reviewed and corrected the final version of the manuscript and also providing useful insight for improving it. All authors contributed to the article and approved the submitted version.

\section{FUNDING}

This study was funded by the Istituto di Ricerca e Cura a Carattere Scientifico (IRCCS) Istituto delle Scienze Neurologiche di Bologna.

\section{ACKNOWLEDGMENTS}

The authors wish to thank Prof. Damir Janigro for his help in proofreading and commenting the manuscript. 


\section{REFERENCES}

1. Riney K, Bogacz A, Somerville E, Hirsch E, Nabbout R, Scheffer IE, et al. ILAE Classification and Definition of Epilepsy Syndromes with Onset at a Variable Age: Position Statement by the ILAE Task Force on Nosology and Definitions. Currently in Review by the ILAE. Available online at: https://www. ilae.org/guidelines/definition-and-classification/proposed-classificationand-definition-of-epilepsy-syndromes/proposed-classification-syndromeswith-onset-at-variable-ages (accessed October 26, 2021).

2. Ormerod JA. On epilepsy, in its relation to ear-disease. Brain. (1883) 6:1. doi: 10.1093/brain/6.1.20

3. Serino A, Heydrich L, Kurian M, Spinelli L, Seeck M, Blanke O. Auditory verbal hallucinations of epileptic origin. Epilepsy Behav. (2014) 31:1816. doi: 10.1016/j.yebeh.2013.12.014

4. d'Orsi G, Tinuper P. The "voices" of joan of arc and epilepsy with auditory features. Epilepsy Behav. (2016) 61:281. doi: 10.1016/j.yebeh.2016.05.008

5. Rice JL. Dostoevsky's medical history : diagnosis and dialectic. Russian Rev. (1983) 42:131-61. doi: 10.2307/129643

6. Swartz CM. Misdiagnosis of schizophrenia for a patient with epilepsy. Psychiatric Services. (2001) 52:109-10. doi: 10.1176/appi.ps521109

7. Hug A, Bartsch A, Gutschalk A. Voices behind the left shoulder: two patients with right-sided temporal lobe epilepsy. J Neurol Sci. (2011) 305:1436. doi: 10.1016/j.jns.2011.03.029

8. Prueter C, Waberski TD, Norra C, Podoll K. Palinacousis leading to the diagnosis of temporal lobe seizures in a patient with schizophrenia. Seizure. (2002) 11:198-200. doi: 10.1053/seiz.2001.0573

9. Phillips HA, Scheffer IE, Berkovic SF, Hollway GE, Sutherland GR, Mulley JC. Localization of a gene for autosomal dominant nocturnal frontal lobe epilepsy to chromosome 20q13.2. Nat Genet. (1995) 10:1178. doi: 10.1038/ng0595-117

10. Ottman R, Risch N, Hauser WA, Pedley TA, Lee JH, Barker-Cummings C, et al. Localization of a gene for partial epilepsy to chromosome 10q. Nat Genet. (1995) 10:56-60. doi: 10.1038/ng0595-56

11. Poza JJ, Sáenz A, Martínez-Gil A, Cheron N, Cobo AM, Urtasun M, et al. Autosomal dominant lateral temporal epilepsy: clinical and genetic study of a large basque pedigree linked to chromosome 10q. Ann Neurol. (1999) 45:182-8. doi: 10.1002/1531-8249(199902)45:2<182::aid-ana8>3.0.co;2-g

12. Winawer MR, Ottman R, Hauser WA, Pedley TA. Autosomal dominant partial epilepsy with auditory features: defining the phenotype. Neurology. (2000) 54:2173-6. doi: 10.1212/wnl.54.11.2173

13. Kalachikov S, Evgrafov O, Ross B, Winawer M, Barker-Cummings C, Boneschi FM, et al. Mutations in LGI1 cause autosomal-dominant partial epilepsy with auditory features. Nat Genet. (2002) 30:335-41. doi: 10.1038/ng832

14. Gu W, Brodtkorb E, Steinlein OK. LGI1 is mutated in familial temporal lobe epilepsy characterized by aphasic seizures. Ann Neurol. (2002) 52:3647. doi: $10.1002 /$ ana. 10280

15. Dazzo E, Fanciulli M, Serioli E, Minervini G, Pulitano P, Binelli $S$, et al. Heterozygous reelin mutations cause autosomaldominant lateral temporal epilepsy. Am J Hum Genet. (2015) 96:992-1000. doi: 10.1016/j.ajhg.2015.04.020

16. Pippucci T, Licchetta L, Baldassari S, Palombo F, Menghi V, Leta C, et al. Epilepsy with auditory features: a heterogeneous clinico-molecular disease. Neurol Genet. (2015) 1:e5. doi: 10.1212/NXG.0000000000000005

17. Dazzo E, Rehberg K, Michelucci R, Passarelli D, Boniver C, Dri VV, et al. Mutations in MICAL-1cause autosomal-dominant lateral temporal epilepsy. Ann Neurol. (2018) 83:483-93. doi: 10.1002/ana.25167

18. Bisulli F, Tinuper P, Avoni P, Striano P, Striano S, d'Orsi G, et al. Idiopathic partial epilepsy with auditory features (IPEAF): a clinical and genetic study of 53 sporadic cases. Brain. (2004) 127:1343-52. doi: 10.1093/brain/awh151

19. Martínez M, Masakazu S, Munari C, Porter R, Roger J, Wolf P. Commission on classification and terminology of the international league against epilepsy. Proposal for revised classification of epilepsies and epileptic syndromes. Epilepsia. (1989) 30:389-99. doi: 10.1111/j.1528-1157.1989.tb05316.x

20. ILAE. Temporal Lobe Seizure. (2020). Available online at: https://www. epilepsydiagnosis.org/seizure/temporal-overview.html (accessed October 26, 2021).

21. Bisulli F, Rinaldi C, Pippucci T, Minardi R, Baldassari S, Zenesini C. Seizure : European journal of epilepsy epilepsy with auditory features : contribution of known genes in 112 patients. Seizure Eur J Epilepsy. (2021) 85:1158. doi: 10.1016/j.seizure.2020.12.015

22. Michelucci R, Pulitano P, Di Bonaventura C, Binelli S, Luisi C, Pasini $\mathrm{E}$, et al. The clinical phenotype of autosomal dominant lateral temporal lobe epilepsy related to reelin mutations. Epilepsy Behav. (2017) 68:1037. doi: 10.1016/j.yebeh.2016.12.003

23. Kobayashi E, Santos NF, Torres FR, Secolin R, Sardinha LAC, LopezCendes I, et al. Magnetic resonance imaging abnormalities in familial temporal lobe epilepsy with auditory auras. Arch Neurol. (2003) 60:154651. doi: 10.1001/archneur.60.11.1546

24. Tessa C, Michelucci R, Nobile C, Giannelli M, Della Nave R, Testoni S, et al. Structural anomaly of left lateral temporal lobe in epilepsy due to mutated LGI1. Neurology. (2007) 69:1298300. doi: 10.1212/01.wnl.0000277045.16688.b6

25. Abarrategui B, Mai R, Sartori I, Francione S, Pelliccia V, Cossu M, et al. Temporal lobe epilepsy: a never-ending story. Epilepsy Behav. (2021) 122:108122. doi: 10.1016/j.yebeh.2021.108122

26. Beffert U, Weeber EJ, Durudas A, Qiu S, Masiulis I, Sweatt JD, et al. Modulation of synaptic plasticity and memory by reelin involves differential splicing of the lipoprotein receptor Apoer2. Neuron. (2005) 47:56779. doi: 10.1016/j.neuron.2005.07.007

27. Chernova OB, Somerville RPT, Cowell JK. A novel gene, LGI1, from 10q24 is rearranged and downregulated in malignant brain tumors. Oncogene. (1998) 17:2873-81. doi: 10.1038/sj.onc.1202481

28. Hivert B, Marien L, Agbam KN, Faivre-Sarrailh C. ADAM22 and ADAM23 modulate the targeting of the Kv1 channel-associated protein LGI1 to the axon initial segment. J Cell Sci. (2019) 132:jcs219774. doi: 10.1242/jcs.2 19774

29. Boillot M, Lee CY, Allene C, Leguern E, Baulac S, Rouach N. LGI1 acts presynaptically to regulate excitatory synaptic transmission during early postnatal development. Sci Rep. (2016) 6:1-9. doi: 10.1038/srep 21769

30. Yamagata A, Fukai S. Insights into the mechanisms of epilepsy from structural biology of LGI1-ADAM22. Cell Mol Life Sci. (2020) 77:26774. doi: 10.1007/s00018-019-03269-0

31. Ohkawa T, Fukata Y, Yamasaki M, Miyazaki T, Yokoi N, Takashima H, et al. Autoantibodies to epilepsy-related LGI1 in limbic encephalitis neutralize LGI1-ADAM22 interaction and reduce synaptic AMPA receptors. J Neurosci. (2013) 33:18161-74. doi: 10.1523/JNEUROSCI.3506-13.2013

32. Van Sonderen A, Thijs RD, Coenders EC, Jiskoot LC, Sanchez E, De Bruijn MAAM, et al. Anti-LGI1 encephalitis: clinical syndrome and long-term follow-up. Neurology. (2016) 87:144956. doi: 10.1212/WNL.0000000000003173

33. Baulac S, Weckhuysen S. DEPDC5-related epilepsy. In: Adam MP, Ardinger $\mathrm{HH}$, Pagon RA, et al., editors. GeneReviews $\AA$. Seattle: University of Washington (2016).

34. Griffith JL, Wong M. The mTOR pathway in treatment of epilepsy: a clinical update. Future Neurol. (2018) 13:49-58. doi: 10.2217/fnl-2018-0001

35. Arcangelo GD, Miao GG, Chent S, Soares HD, Morgan JI, Curran T. A protein related to extracellular matrix proteins deleted in the mouse mutant reeler. Nature. (1995) 374:719-2323. doi: 10.1038/374719a0

36. Chang BS, Duzcan F, Kim S, Cinbis M, Aggarwal A, Apse KA, et al. The role of RELN in lissencephaly and neuropsychiatric disease. Am J Med Genet Part B Neuropsychiatr Genet. (2007) 144:58-63. doi: 10.1002/ajmg.b. 30392

37. Yu NN, Tan MS, Yu JT, Xie AM, Tan L. The role of reelin signaling in Alzheimer's disease. Mol Neurobiol. (2016) 53:5692700. doi: 10.1007/s12035-015-9459-9

38. Leonardi E, Dazzo E, Aspromonte MC, Tabaro F, Pascarelli S, Tosatto SCE, et al. CNTNAP2 mutations and autosomal dominant epilepsy with auditory features. Epilepsy Res. (2018) 139:51-3. doi: 10.1016/j.eplepsyres.2017. 11.006

39. Bisulli F, Licchetta L, Baldassari S, Muccioli L, Marconi C, Cantalupo G, et al. SCN1A mutations in focal epilepsy with auditory features: widening the spectrum of GEFS plus. Epileptic Disord. (2019) 2:18591. doi: 10.1684 /epd.2019.1046

40. Bisulli F, Menghi V, Vignatelli L, Licchetta L, Zenesini C, Stipa C, et al. Epilepsy with auditory features: long-term outcome and predictors 
of terminal remission. Epilepsia. (2018) 59:834-43. doi: 10.1111/epi. 14033

Conflict of Interest: The authors declare that the research was conducted in the absence of any commercial or financial relationships that could be construed as a potential conflict of interest.

Publisher's Note: All claims expressed in this article are solely those of the authors and do not necessarily represent those of their affiliated organizations, or those of the publisher, the editors and the reviewers. Any product that may be evaluated in this article, or claim that may be made by its manufacturer, is not guaranteed or endorsed by the publisher.

Copyright $\odot 2022$ Furia, Licchetta, Muccioli, Ferri, Mostacci, Mazzoni, Menghi, Minardi, Tinuper and Bisulli. This is an open-access article distributed under the terms of the Creative Commons Attribution License (CC BY). The use, distribution or reproduction in other forums is permitted, provided the original author(s) and the copyright owner(s) are credited and that the original publication in this journal is cited, in accordance with accepted academic practice. No use, distribution or reproduction is permitted which does not comply with these terms. 